\title{
Ion-Shading Effects During Metal Etch in Plasma Processing
}

\author{
Tsitsi G. Madziwa-Nussinov, Member, IEEE, Donald Arnush, and Francis F. Chen, Life Fellow, IEEE
}

\begin{abstract}
Self-consistent computations of electric fields (E-fields) and ion orbits inside trenches were done in order to verify Hashimoto's hypothesis of damage that is induced during plasma processing. In his well-accepted theory, Hashimoto proposed a mechanism for electron-shading damage, whereby the photoresist at the tops of trenches and vias collects a negative charge from the thermal electrons, creating an E-field, which prevents electrons from reaching the trench bottom, where collector is located. The sheath E-field accelerates the ions and drives them straight into the trench where they impinge on the collector, and charge it positive if it is isolated. In the computations presented in this paper, it is shown that ion orbits depend only on the E-fields at the entrance and are sensitive to changes in the shape of the photoresist layer there. In addition to the electronshading mechanism, there is an "ion-shading" effect that protects part of the trench walls, and the number of ions that strike the wall is too small to cause any deformation of the walls.
\end{abstract}

Index Terms-Electron-shading damage, ion shading, ion trajectories, metal etch, plasma processing.

\section{INTRODUCTION}

$\mathbf{P}$ LASMA etching [1]-[5] is currently widely used in the fabrication of silicon-based integrated circuits. The process is used to produce high-resolution patterns in many of the thin layers of the circuits and to selectively remove masking layers; it is based on the following sequence of microscopic reaction steps. Electrons are accelerated by dc, RF, or microwave electric fields (E-fields) and collide inelastically with suitable precursor molecules to produce ions, atoms, and radicals. A complex mixture of reactive species is produced. Neutral and ionic reactive species strike the surfaces that are in contact with them to form products that are volatile.

In high density plasma processing, consequences of plasma-surface interactions are to a significant extent controlled by the incident ion fluxes and their energies. An electron-free space-charge region designated as a "sheath" forms between a plasma and a contacting solid surface. Sheaths [2], [6] are of critical importance for plasma etching, since positive ions are accelerated toward the surface when entering

Manuscript received February 15, 2007; revised July 12, 2007.

T. G. Madziwa-Nussinov is with the Los Alamos National Laboratory, Los Alamos, NM 87545 USA (e-mail: tsitsi@ lanl.gov).

D. Arnush, deceased, was with the Electrical Engineering Department, University of California, Los Angeles, CA 90095 USA.

F. F. Chen is with the Electrical Engineering Department, University of California, Los Angeles, CA 90095 USA (e-mail: ffchen@ee.ucla.edu).

Color versions of one or more of the figures in this paper are available online at http://ieeexplore.iee.org.

Digital Object Identifier 10.1109/TPS.2007.905203

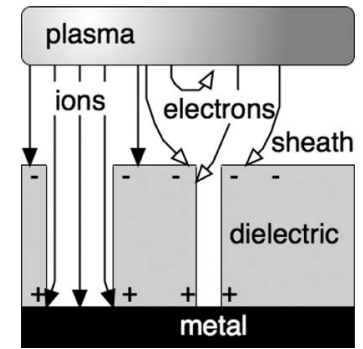

Fig. 1. Illustration of electron shading. The proximity of the plasma's sheath edge is greatly exaggerated.

a sheath. Most of the ion energy is provided by acceleration in the sheath E-fields established by self-biasing the wafer chuck. The accelerated ions bombard the surface with energies that are much greater than thermal energies. Fabrication of high-speed semiconductor circuits depends on etching submicrometer trenches and holes with straight walls, which are guided by sheath-accelerated ions that strike the substrate at a normal angle.

The phenomenon of electron shading, as hypothesized by Hashimoto [7], [8], is illustrated in Fig. 1. The electron-shading effect [9]-[11] is caused by the difference in isotropy of ions and electrons. Electrons enter the sheath with an isotropic angular distribution, while ions, on the other hand, are accelerated in the presheath to the Bohm velocity. Ion velocity in the direction perpendicular to the wafer is larger than the thermal velocity of the ions in the parallel direction. This initial directional difference is greatly amplified as the particles traverse across the sheath. Ions are accelerated, further reaching energies of tens of volts or more. The bombardment of the trench bottom loosens the first few monolayers, allowing the etchant atoms to combine much more effectively than without the ions. At the same time, the electrons, which remain in an isotropic Maxwellian distribution, get decelerated in the sheath, and most of them are returned to the plasma.

Only a small fraction of the electrons crossing the sheath edge will make it to the wafer surface where they charge the nonconductive photoresist to its floating potential. This negative charge allows very few electrons to enter the trench; thus, the ions deposit a positive charge onto the trench bottom [12].

A description of the model, including the assumptions made for simplicity, is given in Section II. In Section III, some of the results are given and discussed. The conclusion is given in Section IV. The bulk of the computational results is given elsewhere [13], [14]. 


\section{MODEL}

\section{A. Model Assumptions and Scale Invariance}

In plasma processing, a planar sheath separates the plasma from the silicon wafer onto which submicrometer circuits are built by deposition and etching. These features have a minimum size (or critical dimension) of $60 \mathrm{~nm}$ or below. The sheath thickness $s$ is at least $5 \lambda_{D}$, where $\lambda_{D}$ is the Debye length, and can be several times larger if a large negative dc bias is applied to the wafer. Taking $n=10^{12} \mathrm{~cm}^{-3}$ and $K T_{e}=1 \mathrm{eV}$ as extreme values that are likely to exist at the sheath edge, it is found that $s$ is, at least, $37 \mu \mathrm{m}$, extending over the 200-300-mm diameter of the wafer. Thus, the sheath is at least 100 times thicker than the feature sizes and orders of magnitude wider. It can therefore be assumed that ions emitted from the sheath edge have straight trajectories normal to the wafer as they approach its surface. As the trenches are much smaller than both the sheath and the Debye lengths, scale invariance justifies the use of the simpler Laplace equation, rather than the Poisson equation, to compute particle trajectories in the model.

To show this justification, we write Poisson's equation as

$$
\varepsilon_{0} \widehat{\nabla}^{2} V=e\left(\widehat{n}_{e}-\widehat{n}_{i}\right)
$$

A system is adopted whereby the capped quantities (such as $\widehat{\nabla}$ ) are in dimensional units and the normal letters are dimensionless. With the usual definitions

$$
\eta \equiv e \widehat{V} / K T_{e}, \quad \lambda_{D}^{2} \equiv \varepsilon_{0} K T_{e} / n_{0} e^{2}, \quad c_{s}^{2} \equiv K T_{e} / M
$$

(1) becomes

$$
\frac{\varepsilon_{0} K T_{e}}{n_{0} e^{2}} \widehat{\nabla}^{2} \eta=\lambda_{D}^{2} \widehat{\nabla}^{2} \eta=\left(\frac{n_{e}}{n_{0}}-\frac{n_{i}}{n_{0}}\right) .
$$

Let $w$ be the scale length of the gradient $\widehat{\nabla}$, and define $\mathbf{r} \equiv \widehat{\mathbf{r}} / w$, so that $\nabla^{2}=w^{2} \widehat{\nabla}^{2}$. This yields

$$
\nabla^{2} \eta=\frac{w^{2}}{\lambda_{D}^{2}}\left(\frac{n_{e}}{n_{0}}-\frac{n_{i}}{n_{0}}\right) \approx 0 .
$$

Although $n_{i} \gg n_{e}$ in the trench, $n_{i} / n_{0}$ is still $<1$. Thus, as long as $w^{2} / \lambda_{D}^{2} \ll 1$, only the dimensionless Laplace's equation

$$
\nabla^{2} \eta=0
$$

needs to be solved, which is subject to the boundary condition $\eta=\eta_{b}\left(\mathbf{r}_{b}\right)$ at $\mathbf{r}=\mathbf{r}_{b}$. The solution would be the same as that of the dimensional problem $\widehat{\nabla}^{2} \eta=0$ with the boundary condition $\eta=\eta_{b}\left(\widehat{r}_{b}\right)$ at $\widehat{r}=\widehat{r}_{b}$. Thus, only the aspect ratio of the trench matters, and not its absolute size, if the Debye length $\lambda_{D}$ is $\gg w$. The space charge deep inside the sheath is negligible.

The ion trajectories are computed from

$$
\frac{d^{2} \widehat{r}}{d t^{2}}=-\frac{e}{M} \widehat{\nabla} V=-\frac{e}{M} \frac{K T_{e}}{e} \nabla \eta=-c_{s}^{2} \widehat{\nabla} \eta .
$$

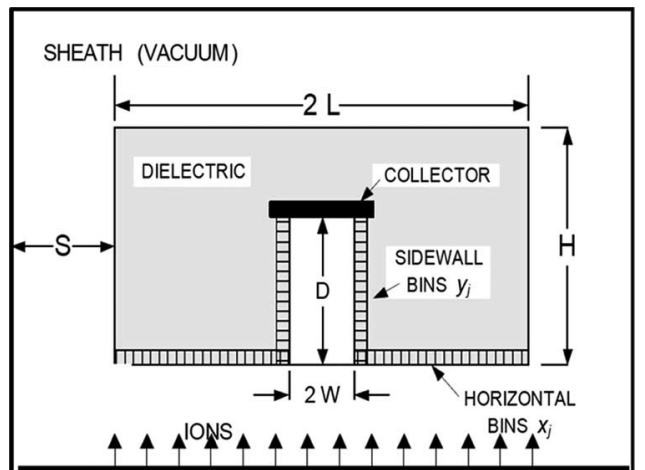

Fig. 2. Computational region. The sheath edge is at the bottom, and the trench is shown inverted, with the collector at the top. The outer region is a vacuum, and the inner region is a dielectric material. Ions are emitted from the sheath edge at the bottom.

In terms of $\mathbf{r}$, this becomes $w^{2} d^{2} \mathbf{r} / d t^{2}=-c_{s}^{2} \nabla \eta$. Defining $\tau \equiv c_{s} t / w$, we have

$$
d^{2} \mathbf{r} / d \tau^{2}=-\nabla \eta
$$

which has the same form as (6), regardless of $w$. Thus, the ion orbits are geometrically the same on any scale; only the time scale is changed. The computations are in these scaleindependent dimensionless units. Collisions are completely negligible, since all mean free paths are longer than $\lambda_{D}$ and, hence, much larger than $s$.

\section{B. Computational Method}

The 2-D region used for the computation is shown in Fig. 2. A block of dielectric with $k \approx 4$ is surrounded by a vacuum sheath region that is bounded by a conductor representing the sheath edge, which is $S$ dimensionless units away. In practice, $S$ is much larger than the feature size, and for computational purposes, its value is not significant. At the bottom of the trench is a conducting "collector," which is shown at the top in this inverted diagram, representing the substrate being etched; the trench grows in the direction of increasing $y$. Ions are accelerated toward the dielectric block by the sheath E-field, and the surface they strike first is normally photoresist. The photoresist surface is divided into cells $x_{j}$, while the trench walls are divided into smaller cells $y_{j}$. The dielectric has width of $2 L=14$ and height of $H=10$, while the trench has width of $2 W$ and depth $D$, with aspect ratio $A_{\mathrm{R}}=D / 2 W$. Ions are injected vertically from the $V \equiv 0$ sheath edge at $y=0$ with the Bohm velocity $c_{s}$. The collector is at an adjustable potential $V_{\mathrm{c}}$.

In all diagrams in this paper, the format is such that the trajectories are inverted as in Fig. 2. The collector is always at the top, and all trench sidewalls are insulating.

Three physically reasonable simplifying assumptions are made: 1) The sheath edge is planar, and monoenergetic ions are ejected at $90^{\circ}$ to it; 2 ) the ion velocity at the sheath edge has the Bohm value $c_{s}$ corresponding to an energy $(1 / 2) K T_{e}$; and 3) the electrons have a Maxwellian distribution everywhere. The last is true if $V_{\mathrm{c}}$ is negative, as is normal for a biased 


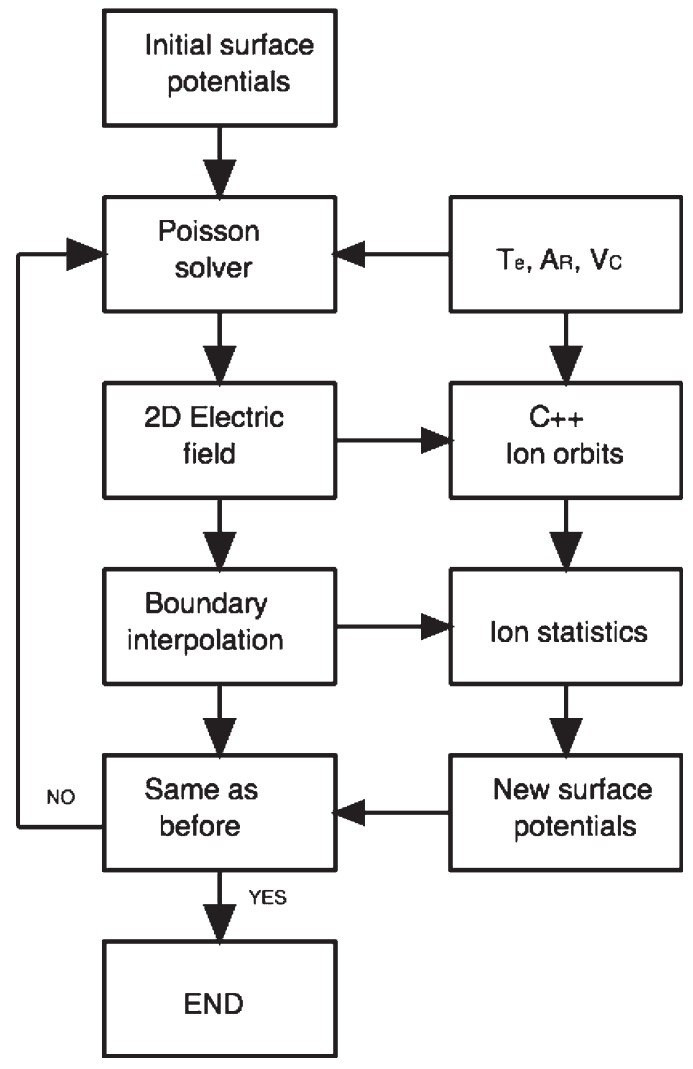

Fig. 3. Computation flowchart.

wafer, so that electrons see a repelling potential everywhere. The Boltzmann relation

$$
n_{e} / n_{s}=\exp \left[\left(V-V_{s}\right) / T_{\mathrm{eV}}\right], \quad T_{\mathrm{eV}} \equiv K T_{e} / e
$$

then holds for any shape. Here, $n_{s}=n_{i}=n_{e}$ at the sheath edge by definition, and we have taken $V_{s}$ to be zero. Thus, the bulk plasma has potential $V=+(1 / 2) T_{\mathrm{eV}}$.

The potential on a floating surface is found by equating the electron and ion fluxes. The electron flux is

$$
\Gamma_{e}=n_{e} \nu_{r}=n_{s} \nu_{r} \exp \left[\left(V-V_{s}\right) / T_{\mathrm{eV}}\right]
$$

where

$$
\nu_{r}=\left(K T_{e} / 2 \pi m\right)^{\frac{1}{2}}
$$

is the random thermal velocity normal to a surface. The ion flux at $y=0$ is simply

$$
\Gamma_{0} \equiv \Gamma_{i}(0)=n_{s} c_{s}=n_{s}\left(K T_{e} / M\right)^{\frac{1}{2}} .
$$

In the absence of a trench, the substrate surface at $y_{j}$ charges to the usual floating potential $V_{\mathrm{f}}$ given by $\Gamma_{i}\left(y_{j}\right)=\Gamma_{i}(0)=\Gamma_{e}$

$$
\left(V_{\mathrm{f}}-V_{s}\right) / T_{\mathrm{eV}}=-\ln (M / 2 \pi m)^{\frac{1}{2}} \approx-4.68 \text { for argon. }
$$

$V_{s}=0$, so that the computation is in a grounded box. Since $V_{s}$ is $\approx-(1 / 2) T_{\mathrm{eV}}$ relative to the plasma, $V_{\mathrm{f}}$ is $\approx-5.18 T_{\mathrm{eV}}$ relative to the plasma or $\approx-15 \mathrm{~V}$ for $K T_{e}=3 \mathrm{eV}$ in argon.

The potential to which each surface element $\Delta x_{j}$ of the photoresist or $\Delta y_{j}$ of the trench wall charges is computed as

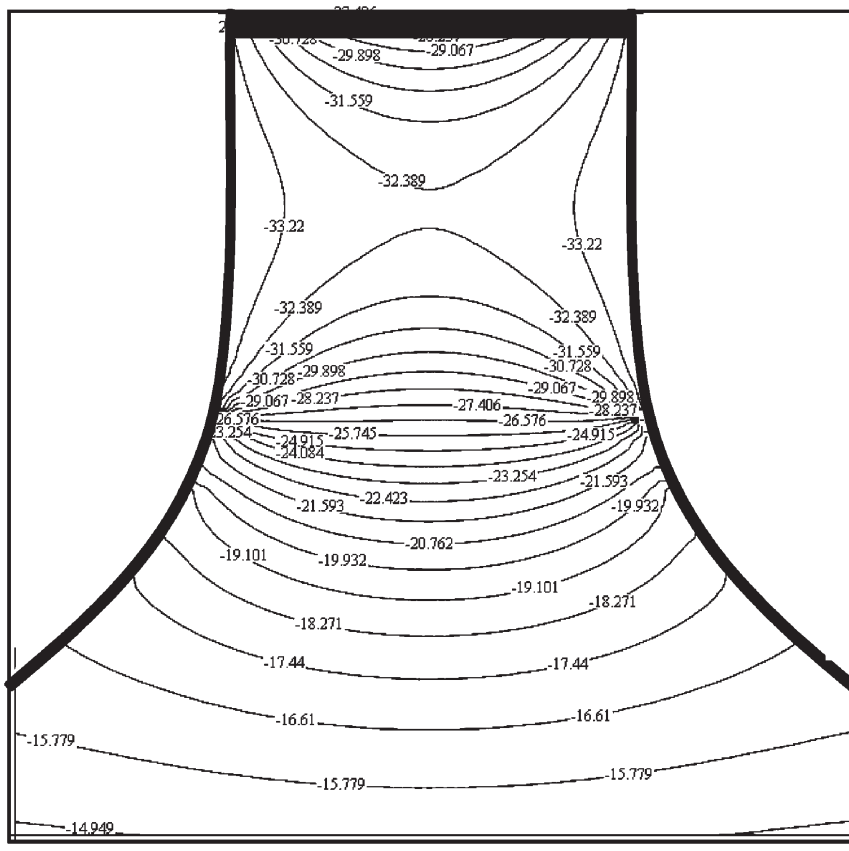

Fig. 4. Typical equipotential lines inside the trench for a collector bias of $-30 \mathrm{~V}$. The dark material is the conducting bottom of the trench.

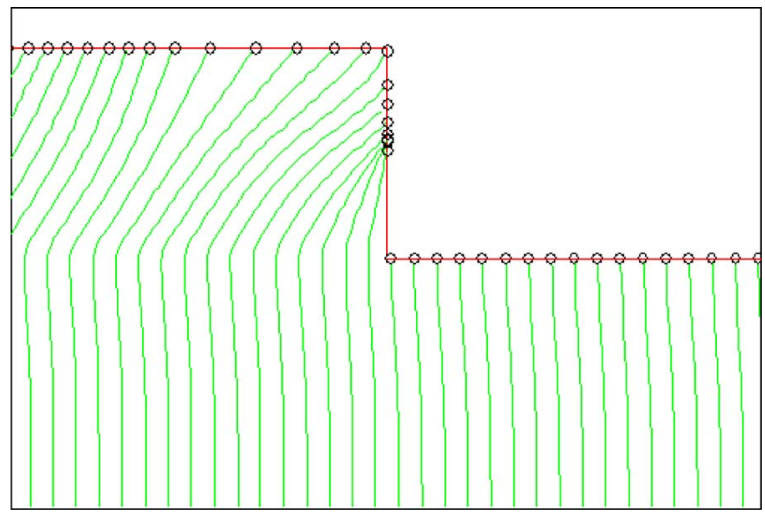

Fig. 5. Example of ion orbit intersections with a boundary. The discrete ion positions usually straddle the boundary and must be interpolated to get the exact position on the boundary.

follows. Let $N$ be the number of ions $\left(\approx 10^{4}\right)$ emitted at $y=0$ over a surface area $L Z$ per unit time, where $Z$ is a length in the ignorable $z$-direction. The emitted ion flux is $\Gamma_{0}=N / L Z=$ $n_{s} c_{s}$. If $N_{j}$ ions strike a surface cell of width $\Delta x_{j}$, the ion flux to that cell is $\Gamma_{i, j}=N_{j} / \Delta x_{j} Z$. The ratio of this to the undisturbed flux $\Gamma_{0}$ is then

$$
R\left(x_{j}\right)=\left(N_{j} / N\right)\left(L / \Delta x_{j}\right)=F\left(x_{j}\right)\left(L / \Delta x_{j}\right)
$$

where $F\left(x_{j}\right)$ is the fraction of all ions that end up in cell $x_{j}$. The electron flux $\Gamma_{e, j}$ to a cell is $n_{s} \nu_{r} \exp \left(V_{j} / T_{\mathrm{eV}}\right)$. Equating this to the ion flux $\Gamma_{i, j}=n_{s} c_{s} R\left(x_{j}\right)$, we have

$$
\nu_{r} e^{V / T_{\mathrm{eV}}}=c_{s} F\left(x_{j}\right) L / \Delta x_{j} .
$$

By using (11), we find the floating potential of that cell relative to the sheath edge to be

$$
V\left(x_{j}\right)=T_{\mathrm{eV}}\left[\ln \left(F_{j} L / \Delta x_{j}\right)-4.68\right]
$$




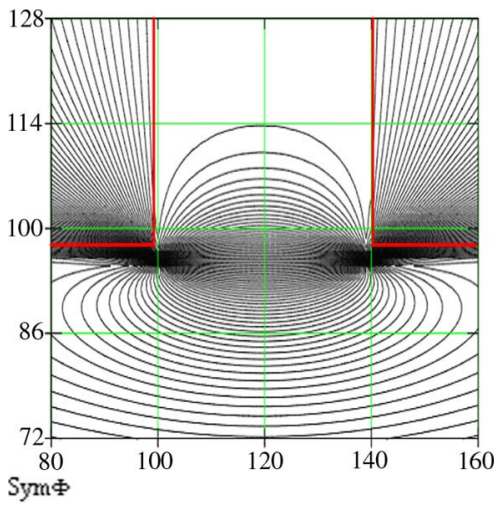

(a)

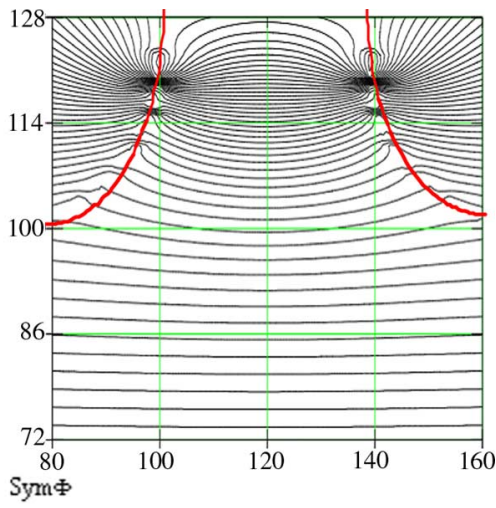

(b)

Fig. 6. Equipotentials near the trench entrance of a sharp and a rounded 2-D geometry.

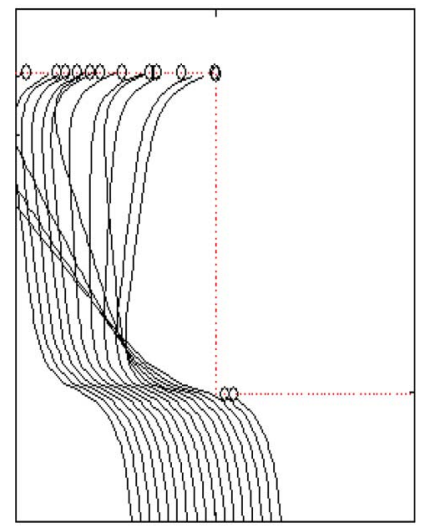

(a)

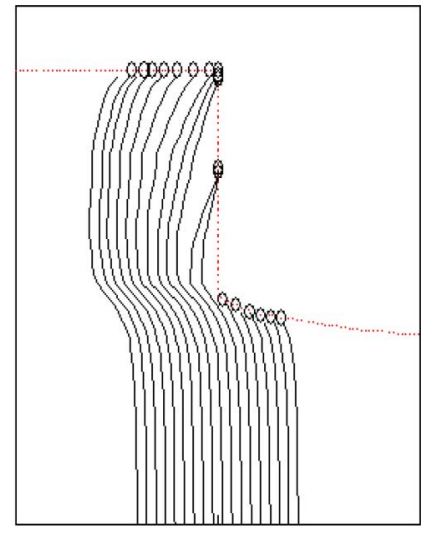

(b)

Fig. 7. Ion orbits in the field of Fig. 6. The horizontal scale has been expanded to show the effect. The thin dotted line shows the trench boundary.

where $F\left(x_{j}\right)$ is found by counting trajectories.

The computational loop is shown in Fig. 3. For a plasma characterized by $K T_{e}$ and ion charge-to-mass ratio, ion orbits are computed first with all insulating surfaces at potential $V_{\mathrm{f}}$ and the collector at potential $V_{\mathrm{c}}$. A Poisson solver [15] with a triangular grid is used to calculate the 2-D E-fields. The timeindependent trajectory of each ion emitted from the sheath edge is then calculated with a $\mathrm{C}++$ program written for this purpose. When the trajectory intersects a surface cell, its contribution to $N_{j}$ is counted. The potential of each cell $V\left(x_{j}\right)$ is then calculated from (14) and used in the first iteration. The ion orbits are then recalculated, giving data for the next iteration. This is continued until $N_{j}$ and $V\left(x_{j}\right)$ converge to steady values. When no ion falls on a cell, (14) diverges. In that case, we assume that the cell actually receives one ion or a fraction of an ion, resulting in $V\left(x_{j}\right) \approx-40 \mathrm{~V}$. The results are not sensitive to this approximation. In some cases, $V\left(x_{j}\right)$ does not converge, but oscillates between two or three patterns after 25 iterations.

At each iteration level, a potential mapping of the whole space is done, and an example is shown in Fig. 4 for a collector bias of -30 V. Fig. 4 shows only the equipotential lines at the entrance and inside the trench. The field rapidly becomes more and more negative toward the trench entrance, and the lines are curved in such a way that ions are drawn into the trench and gain the most momentum at the entrance. Electron trajectories are perpendicular to the field lines.

In this model, we neglect all collisions and surface currents. Surface conductivity was initially accounted for. The effect did not change our result significantly, but rather made our iterations take very long to converge. Because of the scale invariance of the problem, only two parameters need to be set for any given geometry: the aspect ratio $A_{\mathrm{R}}$ and the collector potential $V_{\mathrm{c}}$. The density is irrelevant since the space charge is negligible. The size of the dielectric block does not matter as long as it is big enough.

For definiteness, all dielectrics are given a representative value of $\varepsilon_{\mathrm{R}}=4$. Fig. 5 shows how the ion charges are counted. Since the "time" steps are discrete, an ion trajectory does not necessarily intersect the boundary at the end of a step; the position has to be interpolated. The number of such stopping points in each cell provides the value of $N_{j}$ described above. Once the iteration has converged, the orbits are universal curves for the given values of $A_{\mathrm{R}}, V_{\mathrm{c}}$, and $K T_{e}$. 

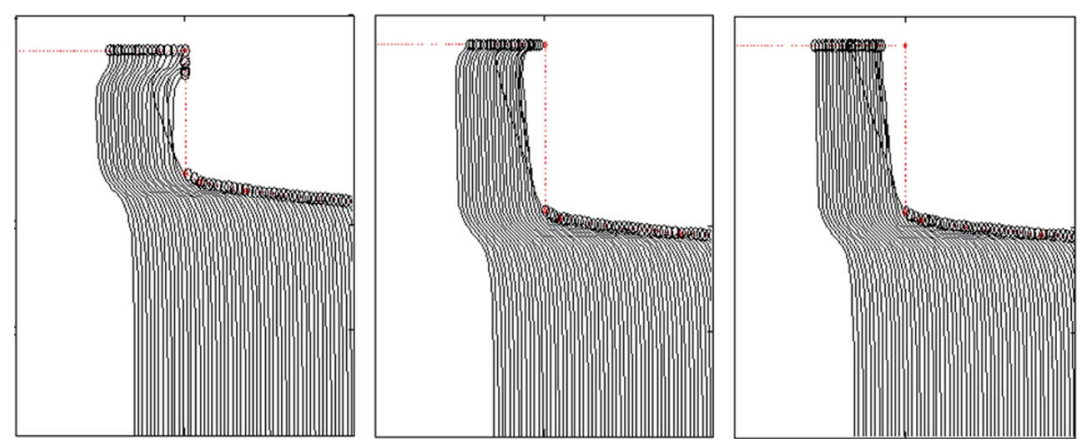

Fig. 8. Ion-shading effect for $A_{\mathrm{R}}=5$ and $V_{\mathrm{c}}=-26 \mathrm{~V}$ (left), $A_{\mathrm{R}}=7$ and $V_{\mathrm{c}}=-26 \mathrm{~V}$ (center), and $A_{\mathrm{R}}=7$ and $V_{\mathrm{c}}=-40 \mathrm{~V}$ (right). The horizontal scale has been expanded.
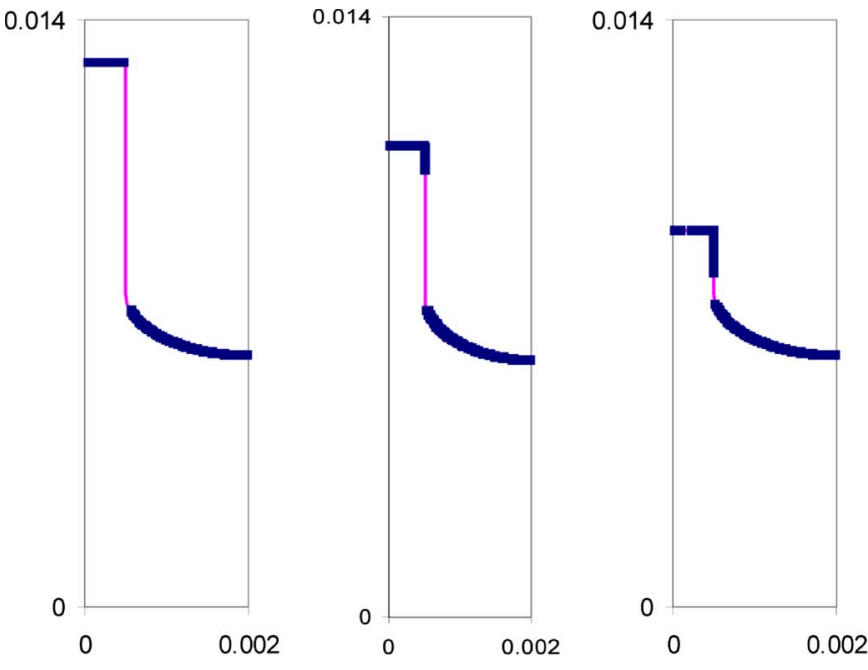

Fig. 9. Ion collection regions in trenches with $A_{\mathrm{R}}=7,5$, and 3 , with $V_{\mathrm{c}}=$ $-26 \mathrm{~V}$. The light regions of the trench wall are not struck by any ions.

\section{RESULTS}

\section{A. Ion Shading}

Fig. 6 shows typical E-field patterns near the trench entrances that are sharp and curved. Trench boundaries are superimposed on the figures. Because of the sharp corners in Fig. 6(a), the field is extremely strong there and causes a large deflection of the ions, as shown in Fig. 7(a). To suppress this unphysical effect, the corners were subsequently rounded into a circular arc. The resulting trajectories [Fig. 7(b)] are more reasonable, but the fact remains that no or very few ions can strike the sidewall near the entrance. This ion shadowing effect, which occurs in addition to the well-known electron-shading effect, is caused by the E-fields outside the trench, which curve the orbits inward. At large negative collector potentials, the entire sidewall can be protected from ion bombardment, as shown in Fig. 8. The decrease in sidewall ions, as the collector potential is increased, was found to be more or less exponential. Physically, the ions gain enough energy at large $\left|V_{\mathrm{c}}\right|$ that the negative sidewalls cannot deflect them.

That ion shading increases with $A_{\mathrm{R}}$ is a more subtle effect. The shaded region along the sidewalls in Fig. 9 shows where the ions fall for three values of $A_{\mathrm{R}}$ at constant $V_{\mathrm{c}}$. The reason that ions are deflected into the sidewalls only for low $A_{\mathrm{R}}$ can be seen from the self-consistent field patterns in Fig. 10. It is seen that the fields are very strong at the ends of the trench, particularly at the corners. At $A_{\mathrm{R}}=7$, the interior of the trench is essentially field-free. Ions are deflected into the sidewall only in the arc region. At $A_{\mathrm{R}}=3$, the fields extend into the interior, where $E_{\mathrm{x}}$ is large enough to impart transverse momentum to the ions streaking in the $y$-direction.

\section{B. Nature of the Ion Trajectories}

The quantitative results can be better understood if one first observes the nature of the ion orbits. Fig. 11 shows three views of the trajectories at $A_{\mathrm{R}}=5$ and $V_{\mathrm{c}}=-26 \mathrm{~V}$. Fig. 11(a) shows the orbits on a normal $x-y$ scale. The ions enter the trench at high velocity, and their deflections are so small that they cannot be seen on this scale. In Fig. 11(b), the horizontal scale has been expanded by a factor of 20 . Here, it can be seen that the ions enter the trench at an angle due to the external E-field. No ions strike the first part of the wall, which is shaded. The negative charges on the wall eventually deflect the ions outward, causing them to strike the corner of the trench. In Fig. 11(c), the horizontal scale has been stretched by a factor of 80 , and intermediate orbits have been plotted, which are shown by the thin lines. Orbits that pass near the corner of the photoresist actually cross and take shapes that are sensitive to the strong fields at the entrance corner. Note that the corner is actually circular, but looks sharp only on this 80-to-1 scale. The pulling of ions into the trench by the charge on the photoresist is shown more clearly in Fig. 12. Incident ions (indicated by the solid lines), headed for the photoresist surface outside the trench, are drawn into the trench by the fields in the arc region. The trajectories show that the ions are bent away from the corner of the photoresist instead of toward it. In the first iteration of the computation, ions land in the corner. The ions landing there then change the surface charge. The selfconsistent surface charge then bends the trajectories the way they are shown, consistently with the equipotential curves in Figs. 4, 6, and 10).

\section{Effect of Photoresist Shape}

As etching progresses, the photoresist will change its shape at the trench entrance, and this will affect the ion orbits. Since the E-fields are strongest there, we expect that the ion orbits would be sensitive to these changes. First, consider the change from 


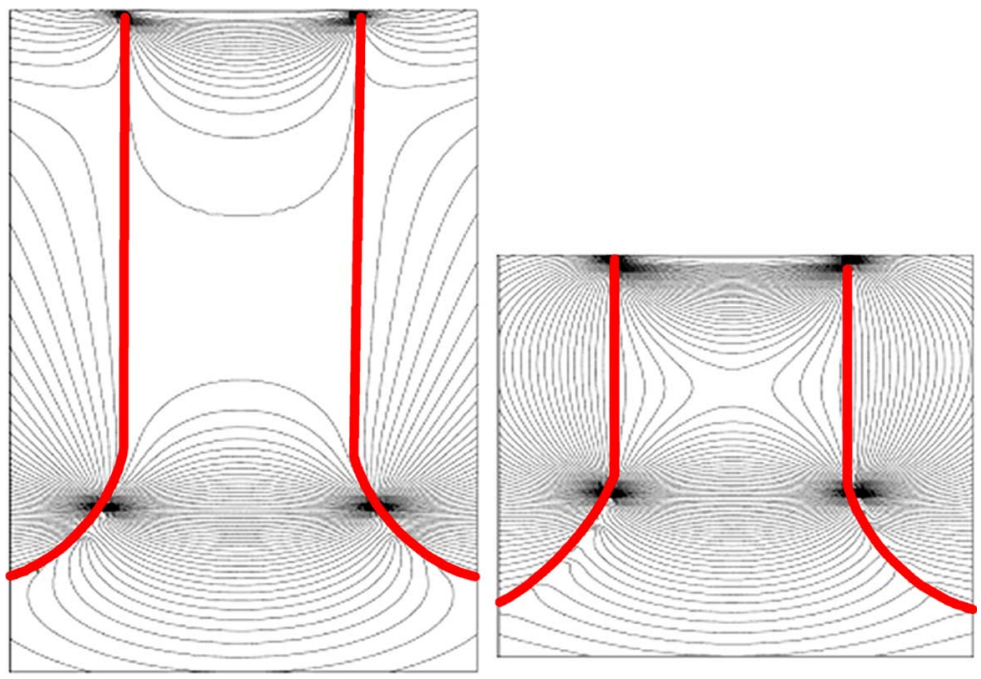

Fig. 10. Field patterns (equipotentials) for $A_{\mathrm{R}}=7$ (left) and $A_{\mathrm{R}}=3$ (right), at $V_{\mathrm{c}}=-22 \mathrm{~V}$.
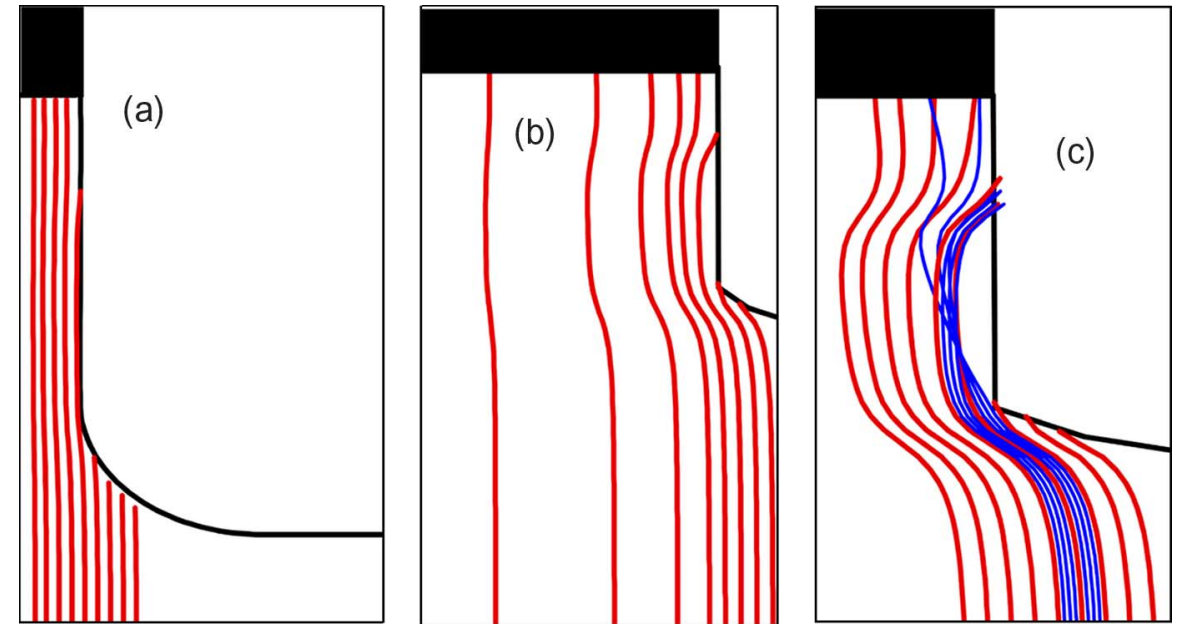

Fig. 11. Ion orbits for $A_{\mathrm{R}}=5$ and $\left|V_{\mathrm{c}}\right|=26 \mathrm{~V}$ with the horizontal scale (a) normal, (b) expanded by a factor of 20, and (c) expanded by a factor of 80 relative to the vertical scale. The orbit spacing has been decreased near the wall for clarity.

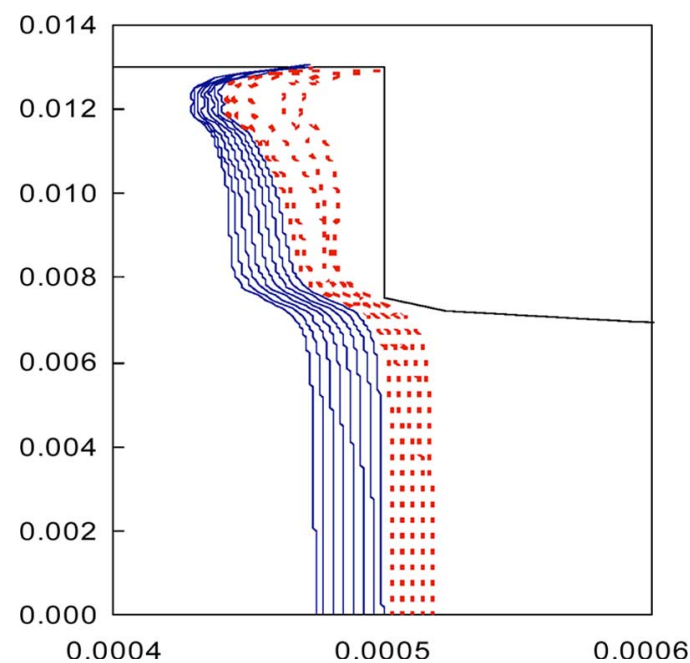

Fig. 12. Ion orbits near the trench edge for $A_{\mathrm{R}}=7$ and $V_{\mathrm{c}}=22$.

a mathematically sharp edge to the rounded edge used in our calculations. This was originally done to improve the convergence of the iterations, but the change in orbits was significant.
TABLE I

ION Distributions With SHARP AND ROUNDED CORNERS

\begin{tabular}{|r|c|c|}
\hline $\boldsymbol{A}_{\mathbf{R}}=\mathbf{7}, \boldsymbol{V}_{\mathbf{c}}=\mathbf{- 1 8 V}$ & $\begin{array}{c}\text { Case A: } \\
\text { Sharp corner }\end{array}$ & $\begin{array}{c}\text { Case B: } \\
\text { Rounded corner }\end{array}$ \\
\hline Collector & $7.9 \%$ & $9.9 \%$ \\
\hline Sidewalls & $0.5 \%$ & $0.3 \%$ \\
\hline Arc & N/A & $20.7 \%$ \\
\hline Flat surface & $91.6 \%$ & $69.1 \%$ \\
\hline $\boldsymbol{A}_{\mathbf{R}}=\mathbf{7 ,} \boldsymbol{V}_{\mathbf{c}}=\mathbf{- 2 6 V}$ & & \\
\hline Collector & $8.2 \%$ & $10.1 \%$ \\
\hline Sidewalls & $0.18 \%$ & $0.00 \%$ \\
\hline
\end{tabular}

Table I shows the percentage of ions landing on each part of the trench for a sample of about $10^{4}$ ions emitted from the entire sheath area. On the photoresist surface, including the arc region, the potential is solved self-consistently, as it was on the trench walls.

Table I also shows that fewer ions reach the collector if the corner is sharp rather than rounded. This is because the sharp 


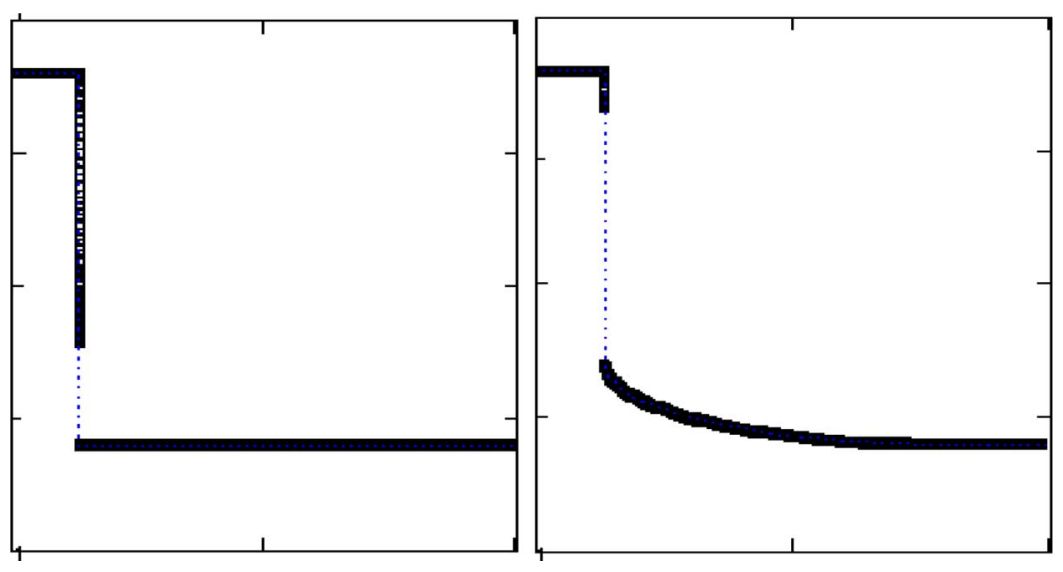

Fig. 13. Distribution of ion collection for sharp and rounded trench entrances. The ion statistics for these figures are shown in Table I.
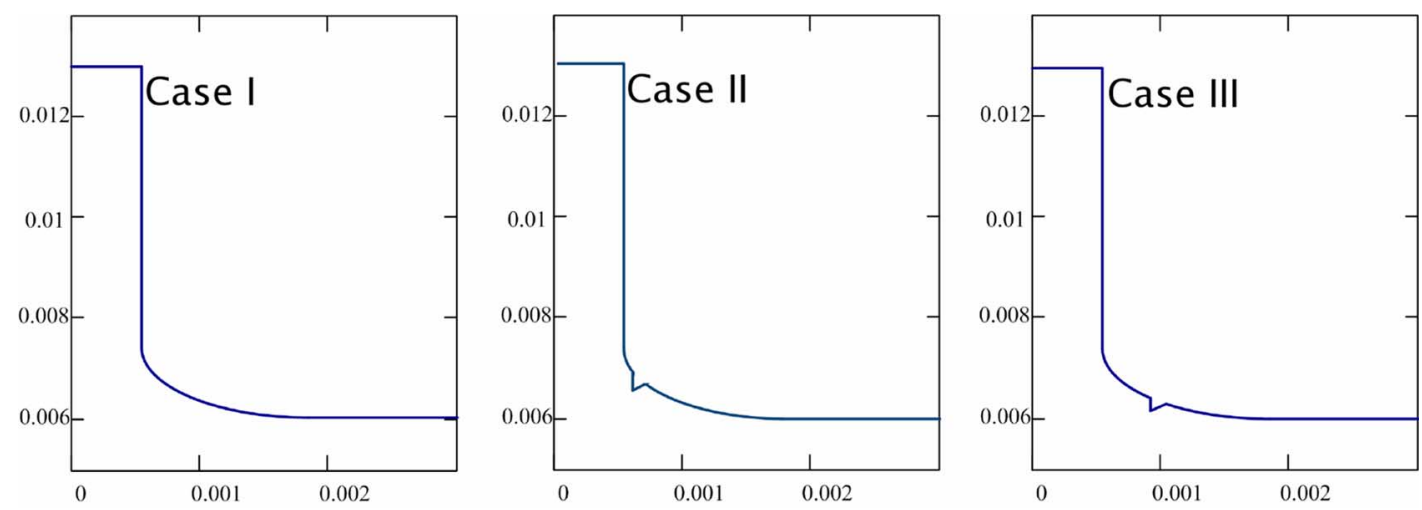

Fig. 14. Profiles of trench entrances without and with bumps.

TABLE II

ION DisTRIBUTIONS WiTh SMALL Bumps

\begin{tabular}{|r|c|c|c|}
\hline $\begin{array}{c}\boldsymbol{A}_{\mathbf{R}}=\mathbf{7}, \\
\boldsymbol{V}_{\mathbf{c}}=\mathbf{- 1 8 V}\end{array}$ & $\begin{array}{c}\text { Case I: } \\
\text { No bump }\end{array}$ & $\begin{array}{c}\text { Case II: } \\
\text { Close bump }\end{array}$ & $\begin{array}{l}\text { Case III: } \\
\text { Far bump }\end{array}$ \\
\hline Collector & $9.9 \%$ & $8.6 \%$ & $9.0 \%$ \\
\hline Sidewalls & $0.3 \%$ & $0.0 \%$ & $1.8 \%$ \\
\hline Arc & $20.7 \%$ & $17.7 \%$ & $17.4 \%$ \\
\hline Flat surface & $69.1 \%$ & $73.7 \%$ & $71.8 \%$ \\
\hline
\end{tabular}

corner more effectively shields ions approaching the trench at an angle. In either case, the fraction of ions hitting the sidewall is extremely small and cannot cause the trench profile defects that have been observed. As expected, the collector current increases, and sidewall current decreases at the higher $\left|V_{\mathrm{c}}\right|$ of $26 \mathrm{~V}$. The difference between cases A and B is even more noticeable in the distribution of ions shown in Fig. 13. In case $\mathrm{A}$, ions are collected over a large part of the sidewall, while in case $\mathrm{B}$, ions are shielded from all but the last bin.

To see the effect of small changes to the entrance shape, small bumps were added onto the arc region in two locations. The statistics are shown in Table II for a sample of 50000 ions. In case III, the bump is farther from the trench than in case II, as shown in Fig. 14. The sidewall ion distributions are shown in the histograms in Fig. 15. It is clear that even a small deformation of the photoresist will change the ion orbits drastically. The self-consistent equipotential lines for these three cases are shown in Fig. 16. In case I, the E-field is very strong near the entrance. The ions are given a kick there and then coast to the trench bottom (at the top in the figure) through the nearly field-free trench. The collector is given only a small bias in this example, so that ions are not strongly drawn into it; some of them hit the sidewall near the bottom, giving rise to E-fields near the bottom corner. In case II, the bump shadows part of the arc region, and the negative surface charge in the shadow gives the ions an extra kick, causing them all to land on the collector, and thus, no sidewall ions. In case III, the bump is back far enough that the ions are deflected into the arc region, lowering the field there. Having undergone less acceleration, the ions are drawn by transverse fields into the sidewall. Nonetheless, the top part of the sidewall is still shadowed. This sensitivity to the exact shape of the photoresist means that the ion orbits change during the etch and cannot be predicted. Fortunately, the ion flux to the sidewalls is in every case so small compared with that on the collector that it cannot significantly affect the quality of the etch.

\section{Summary AND CONCLUSION}

By reducing the problem to a simple dimensionless form, universal curves were obtained for ion trajectories in etched trenches, self-consistently with the sidewall potentials. 

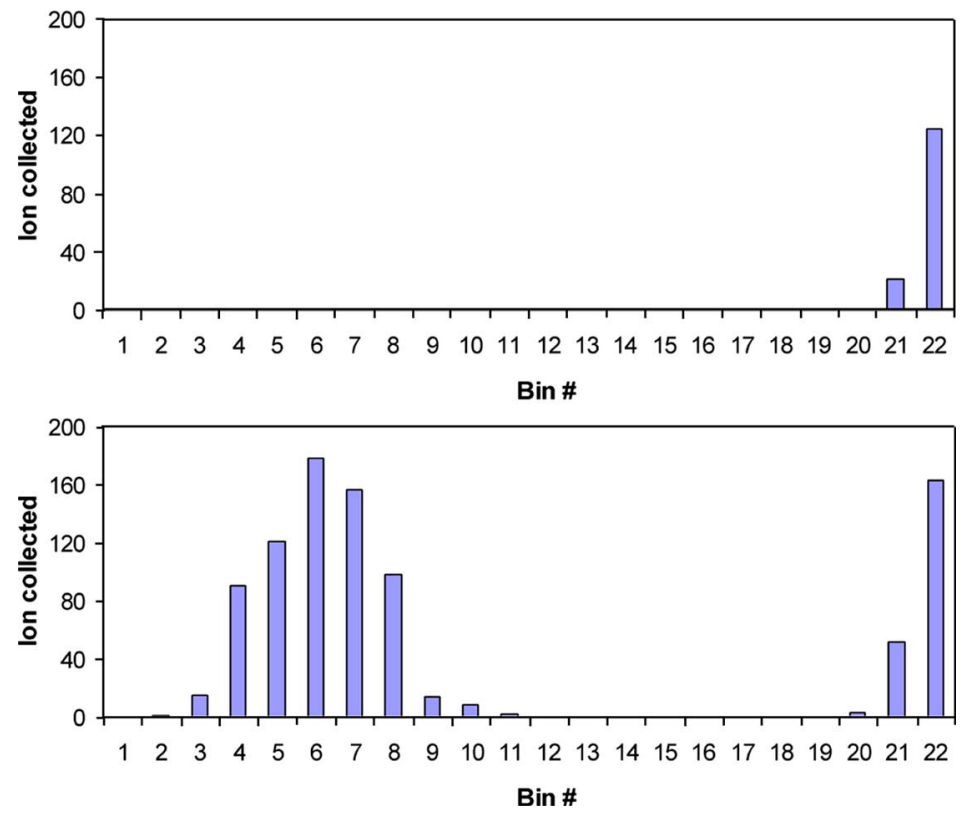

Fig. 15. Sidewall ions location for cases I and III. No ions were collected on the sidewalls for II.
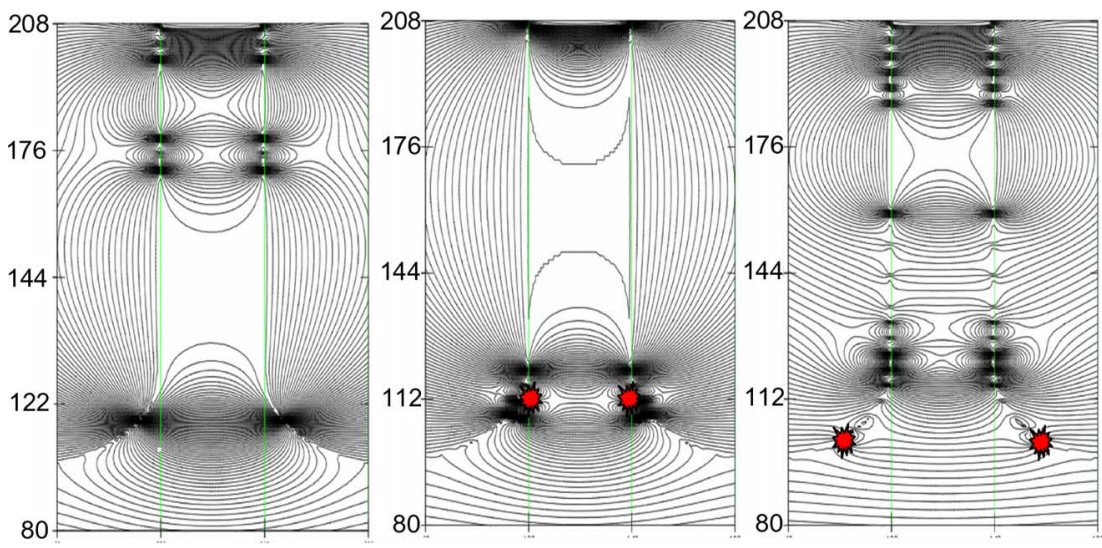

Fig. 16. Field patterns for the profiles shown in Fig. 14.

Reflection of ions from the walls was ignored. Results depend on only two parameters: the aspect ratio $A_{\mathrm{R}}$, and the potential $V_{\mathrm{c}}$ at the trench bottom. Principal findings are as follows.

1) E-fields that are external to the trench cause the ions to enter the trench at an angle. This causes an ion-shading effect, which protects the top part of the trench, and sometimes all of it, from ion bombardment.

2) Ion orbits are determined mainly by the strong fields at the corners of the entrance; they then coast through the trench with little additional deflection.

3) In spite of the strong fields at the entrance, ion orbits are nearly straight and vertical. Therefore, few ions strike the sidewall that they cannot cause deformations of the trench profile.

4) The small ion flux to the walls is very sensitive to the exact shape of the photoresist at the top. This will change in an unpredictable way during the etching process.

5) Contrary to intuition, a deep trench with large $A_{\mathrm{R}}$ will have an insignificant number of ions striking the wall.
These observations differ from commonly held conceptions of how the ion flux behaves in reactive ion etching. By examining the variation with $A_{\mathrm{R}}$, one can get an idea of the changes in ion behavior as the etch progresses. By varying $V_{\mathrm{c}}$, one can gauge the changes during an RF cycle. However, we must note that findings of this paper do not take into consideration the effect of specific plasma chemistry, but are rather generalized to any ions present in the plasma.

\section{REFERENCES}

[1] M. A. Lieberman and A. J. Lichtenberg, Principles of Plasma Discharges and Materials Processing. New York: Wiley, 1994.

[2] G. S. Oehrlein, M. F. Doemling, B. E. E. Kastenmeier, P. J. Matsuo, N. R. Rueger, M. Schaepkens, and T. E. F. M. Standaert, "Surface science issues in plasma etching," IBM J. Res. Develop., vol. 43, no. 1/2, pp. 181198, 1999.

[3] S. M. Sze, Physics of Semiconductor Devices. New York: Wiley, 1981.

[4] M. Armacost, P. D. Hoh, R. Wise, W. Yan, J. J. Brown, J. H. Keller, G. A. Kaplita, S. D. Halle, K. P. Muller, M. D. Naeem, S. Srinivasan, H. Y. Ng, M. Gutsche, A. Gutmann, and B. Spuler, "Plasma-etching processes for ULSI semiconductor circuits," IBM J. Res. Develop., vol. 43, no. 1/2, pp. 39-72, 1999. 
[5] T. Arikado et al., "Single silicon etching profile simulation," Jpn. J. Appl. Phys., vol. 27, pt. 1, no. 1, pp. 95-99, Jan. 1988.

[6] F. F. Chen, Plasma Physics and Controlled Fusion. New York: Plenum, 1990.

[7] K. Hashimoto, "New phenomena of charge damage in plasma etching: Heavy damage only through dense-line antenna," Jpn. J. Appl. Phys., vol. 32, no. 12B, pp. 6109-6113, Dec. 1993.

[8] K. Hashimoto, "Charge damage caused by electron shading effect," Jpn. J. Appl. Phys., vol. 33, no. 10, pp. 6013-6018, Oct. 1994.

[9] N. Fujiwara, T. Maruyama, and M. Yoneda, "Profile control of polySi etching in electron cyclotron resonance plasma," Jpn. J. Appl. Phys., vol. 34, no. 4B, pp. 2095-2100, Apr. 1995.

[10] G. S. Hwang and K. P. Giapis, "On the link between electron shadowing and charging damage," J. Vac. Sci. Technol. B, Microelectron. Process. Phenom., vol. 15, no. 5, pp. 1839-1842, Sep. 1997.

[11] K. P. Giapis and G. S. Hwang, "Pattern-dependent charging and the role of electron tunneling," Jpn. J. Appl. Phys., vol. 37, no. 4B, pp. 2281-2290, Apr. 1998.

[12] R. Wise, S. Panda, S. Mathad, and R. Ranade, "Observations of patterndependent plasma charging and polymer deposition during deep trench dry etch," Future Fab Int., vol. 11, Jun. 29, 2001.

[13] T. G. Madziwa-Nussinov, "The role of ions in charging damage during high density plasma etching," M.S. thesis, UCLA, Los Angeles, CA, 2003.

[14] T. G. Madziwa-Nussinov, D. Arnush, and F. F. Chen, "Ion orbits in plasma etching of semiconductors," Phys. Plasmas, (to be published).

[15] TriComp E-Stat 6.0, and TriComp Mesh 6.0 Codes. Copyright Field Precision. [Online]. Available: http://www.fieldp.com

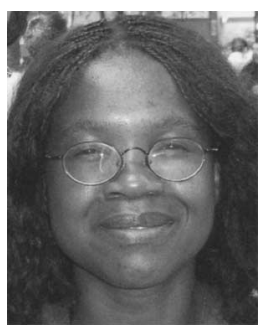

Tsitsi G. Madziwa-Nussinov (M'99) was born in Zimbabwe. She received the B.Sc. Hons. degree in physics from the University of Zimbabwe, Harare, Zimbabwe, in 1994 and the M.S. degree in physics and the Ph.D. degree in electrical engineering from the University of California, Los Angeles, in 1997 and 2003, respectively.

Since 2005, she has been with the Plasma Physics group at Los Alamos National Laboratory, Los Alamos, NM, doing experiments on the reconnection scaling experiment, where she is currently a Postdoctoral Fellow working on plasma thruster experiments.
Donald Arnush, was a Group Leader with TRW Inc., Redondo Beach, CA, for many years. After retirement, until his death, he was an Adjunct Professor with the Electrical Engineering Department, University of California, Los Angeles.

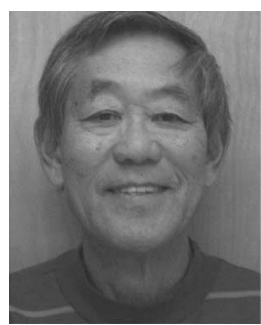

Francis F. Chen (SM'72-LF' 80) received the Ph.D. degree from Harvard University, Cambridge, MA, in 1954 .

$\mathrm{He}$ is a Plasma Physicist with a career extending over 52 years and encompassing both experiment and theory. He has devoted about a decade each to the subfields of magnetic fusion, laser fusion, plasma diagnostics, basic plasma physics, and lowtemperature plasma physics. He is the author of the textbook Introduction to Plasma Physics and Controlled Fusion. His current research interest includes plasma processing of semiconductor circuits, particularly the radio-frequency sources used to make computer chips, and the physical processes that permit etching millions of transistors on a single chip. Although formally retired from teaching, he still maintains an Active Research Group, Electrical Engineering Department, University of California, Los Angeles. 